\title{
The CRISPR growth spurt: from bench to clinic on versatile small RNAs
}

\begin{abstract}
Clustered Regulatory Interspaced Short Palindromic Repeats (CRISPR) in association with CRISPR associated protein (Cas) is an adaptive immune system, playing a pivotal role in the defense of bacteria and archaea. Ease of handling and cost effectiveness make CRISPR-Cas system an ideal programmable nuclease tool. Recent advances in understanding the CRISPRCas system have tremendously improved its efficiency. For instance, it is possible to recapitulate the chronicle CRISPR-Cas from its infancy and inaugurate a developed version by generating novel variants of Cas proteins, subduing off-target effects and optimization of innovative strategies. In summary, CRISPR-Cas system could be employed in a number of applications including providing model systems, rectification of detrimental mutations, and antiviral therapies
\end{abstract}

Keyword: CRISPR-Cas system; DNA repair; Adoptive immunity; Genome editing 